\title{
Biochemical and Functional Interaction of Disrupted-in-Schizophrenia 1 and Amyloid Precursor Protein Regulates Neuronal Migration during Mammalian Cortical Development
}

\author{
Tracy L. Young-Pearse, ${ }^{1}$ Seiyam Suth, ${ }^{1}$ Eric S. Luth, ${ }^{1}$ Akira Sawa, ${ }^{2}$ and Dennis J. Selkoe ${ }^{1}$ \\ ${ }^{1}$ Center for Neurologic Diseases, Brigham and Women's Hospital and Harvard Medical School, Boston, Massachusetts 02115, and ${ }^{2}$ Department of \\ Psychiatry, The Johns Hopkins University, Baltimore, Maryland 21287
}

\begin{abstract}
Although clinically distinct, schizophrenia and Alzheimer's disease are common and devastating disorders that profoundly impair cognitive function. For Alzheimer's disease, key mechanistic insights have emerged from genetic studies that identified causative mutations in amyloid precursor protein (APP) and presenilin. Several genes have been associated with schizophrenia and other major psychoses, and understanding their normal functions will help elucidate the underlying causes of these disorders. One such gene is disrupted-in-schizophrenia 1 (DISC1). DISC1 and APP have been implicated separately in cortical development, with each having roles in both neuronal migration and neurite outgrowth. Here, we report a previously unrecognized biochemical and functional interaction between DISC1 and APP. Using in utero electroporation in the living rat brain, we show that DISC1 acts downstream of APP and Disabled-1 to regulate cortical precursor cell migration. Specifically, overexpression of DISC1 rescues the migration defect caused by a loss of APP expression. Moreover, knockdown of APP in cultured embryonic neurons results in altered subcellular localization of DISC1. Using transfected cells and normal brain tissue, we show that APP and DISC1 coimmunoprecipitate and that the intracellular domain of APP interacts with the N-terminal domain of DISC1. Based on these findings, we hypothesize that the APP cytoplasmic region transiently interacts with DISC1 to help regulate the translocation of DISC1 to the centrosome, where it plays a key role in controlling neuronal migration during cortical development.
\end{abstract}

\section{Introduction}

Schizophrenia and Alzheimer's disease (AD) are two common and devastating disorders that result in impaired cognition and deficiencies in sensory information processing. Although both diseases primarily affect the cerebral cortex and hippocampus, the etiologies and phenotypes of the diseases are clearly distinct. Schizophrenia is a mental disorder of young adulthood with evidence of a neurodevelopmental origin (for review, see JaaroPeled et al., 2009), whereas AD is a neurodegenerative disorder with a typical onset late in life. Here, we report an unexpected interaction between disrupted-in-schizophrenia 1 (DISC1) and $\beta$-amyloid precursor protein (APP), linking key protein factors of schizophrenia and $\mathrm{AD}$ in the process of normal cortical development.

APP is a type I transmembrane glycoprotein expressed throughout the developing and adult brain (for review, see De Strooper and Annaert, 2000). APP is proteolytically processed to release $\mathrm{A} \beta$ (amyloid- $\beta$ protein), a pathological hallmark of $\mathrm{AD}$.

Received March 21, 2010; revised May 11, 2010; accepted May 20, 2010.

This work was supported by National Institutes of Health Grants K99 MH85004 (T.L.Y.-P.) and R01 AG06173 (D.J.S.). We thank J. Hegde and W. Cavanaugh for technical assistance, N. Brandon for a DISC1 antibody, and members of the Selkoe Laboratory for helpful discussions.

Correspondence should be addressed to either Dennis J. Selkoe or Tracy L. Young-Pearse at the above address. E-mail: dselkoe@rics. bwh.harvard.edu or tyoung@rics.bwh.harvard.edu.

DOI:10.1523/JNEUROSCI.1445-10.2010

Copyright $\odot 2010$ the authors $\quad 0270-6474 / 10 / 3010431-10 \$ 15.00 / 0$
Previously, we explored the endogenous function of APP in the developing brain by acutely knocking down APP in cortical precursor cells. These experiments illuminated a role for APP in the migration of neuronal precursor cells (Young-Pearse et al., 2007). Disabled-1 (DAB1), a key neuronal migration factor, was found to act downstream of APP in this process (Young-Pearse et al., 2007). DAB1 phosphorylation in response to Reelin signaling is well established to be necessary for proper cortical cell migration (for review, see Tissir and Goffinet, 2003). Beyond these findings, the pathway through which APP-DAB1 complexes help regulate migration is unknown.

DISC1 was identified through the cloning of the gene disrupted by a balanced translocation of chromosome 1 in a Scottish pedigree with schizophrenia and other major mental illnesses (Millar et al., 2000). Interestingly, a recent genome-wide association study has shown a significant association between a singlenucleotide polymorphism in the intron of DISC1 and late-onset AD (Beecham et al., 2009). DISC1 has been reported to have several functions in the developing nervous system, including roles in neuronal migration (Kamiya et al., 2005), proliferation (Mao et al., 2009), and neurite outgrowth (Miyoshi et al., 2003; Ozeki et al., 2003; Kamiya et al., 2005, 2006). DISC1 has been shown to localize to multiple subcellular compartments and have $>100$ apparent binding partners (Ishizuka et al., 2006; Brandon, 2007). Therefore, DISC1 may act as a scaffold, and its functions in subsets of neurons at distinct times in brain development may be determined by its binding partners and subcellular localization. 
Since the correct migration of neurons affects brain morphology and connectivity, migration defects may underlie certain neurodevelopmental disorders, including schizophrenia. Defining the mechanisms by which DISC1 regulates neuronal migration is a key goal. Here, we demonstrate a previously unrecognized functional interaction between APP and DISC1 in neuronal migration. Furthermore, we show that APP and DISC1 can physically interact in the brain and that a loss of APP causes a subcellular redistribution of the DISC1 protein in primary cortical neurons.

\section{Materials and Methods}

Plasmids. Generation of short hairpin RNA (shRNA) constructs for APP and DAB1 (Young-Pearse et al., 2007), and control shRNA (YoungPearse et al., 2007) and DISC1 (Kamiya et al., 2005), were previously described and characterized. The generations of the following constructs were previously described: hemagglutinin (HA)-tagged human DISC1 (hDISC1)-L, -Lv, 1-402, 403-854, DISC1 NDEL1 (DISC1 lacking the NDEL1 binding site) and DISC1 $\triangle$ SA (DISC1 lacking a self-association site), HA-tagged mouse DISC1, and NDEL1-myc (Kamiya et al., 2005), Itg $\beta 1$-FLAG, FLAG-APP, and APLP1-FLAG (Young-Pearse et al., 2008), FLAG-APP $\triangle$ CT, and APPNATA (Young-Pearse et al., 2007) and NPR3FLAG (Hemming et al., 2008). APP666 encodes human APP from amino acids 1-666 and APP680 encodes amino acids 1-680 (695 splice variant numbering for both). DISC1-S and -Es were cloned by PCR and sequenced to confirm amino acid identity with entries for these human DISC1 splice variants in GenBank (National Center for Biotechnology Information).

In utero electroporation. Sprague Dawley rats (Charles River Laboratories) were housed and cared for under the guidelines established by Harvard University's Institutional Animal Care and Use Committees in compliance with federal standards. Timed-pregnant female rats at embryonic day 15.5 (E15.5) were anesthetized with ketamine/xylazine (100/10 mixture; $0.1 \mathrm{mg} / \mathrm{g}$ body weight, i.p.). The uterine horns were exposed, and a lateral ventricle of each embryo (both males and females) were injected with DNA constructs and Fast Green $(2 \mathrm{mg} / \mathrm{ml}$; SigmaAldrich) via a microinjector (Picospritzer III; General Valve) and pulled glass capillaries. For characterization of shRNA phenotypes, 1.0-1.5

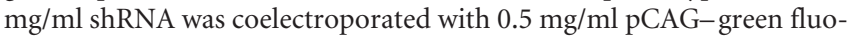
rescent protein (GFP). For rescue experiments, $0.5 \mathrm{mg} / \mathrm{ml}$ shRNA was coelectroporated with $0.5 \mathrm{mg} / \mathrm{ml} \mathrm{pCAG-GFP}$ and $3.0 \mathrm{mg} / \mathrm{ml}$ rescue constructs. All rescue constructs were expressed in the pCAGGs vector. Electroporation was accomplished by discharging a $500 \mathrm{mF}$ capacitor charged to 50-100 V with a sequencing power supply or with a BTX square wave electroporator, at $50-75 \mathrm{~V}$, for $50 \mathrm{~ms}$ on followed by $950 \mathrm{~ms}$ off for five pulses. The voltage was discharged across copper alloy oval plates placed on the uterine wall across the head of the embryo. Brains from rat embryos were harvested $72 \mathrm{~h}$ after electroporation in $4 \%$ paraformaldehyde by immersion. For each plasmid combination, at least three independent brains were analyzed. For 5-bromodeoxyuridine (BrdU) studies, $1 \mathrm{ml}$ of a $10 \mathrm{mg} / \mathrm{ml} \mathrm{BrdU}$ solution was injected intraperitoneally $24 \mathrm{~h}$ after in utero electroporation.

Cell lines and Western blot analysis. COS cells (monkey kidney cell line) were transiently transfected using Lipofectamine 2000 (Invitrogen) in 10 $\mathrm{cm}$ dishes in duplicate with listed constructs. Cells were lysed after $48-72$ $\mathrm{h}$ in $1 \%$ Nonidet P-40 (NP-40) STEN buffer [150 mm sodium chloride, $50 \mathrm{~mm}$ Tris, $2 \mathrm{~mm}$ EDTA, and 1.0\% (v/v) NP-40]. Lysates were electrophoresed on $10-20 \%$ Tricine gels (Invitrogen) or $4-12 \%$ Bis-Tris NuPage gels (Invitrogen) and transferred to nitrocellulose. Western blotting was performed with anti-FLAG M2 (1:1000; Sigma-Aldrich), anti-APP antibodies: C9 (1:1000; Selkoe Laboratory), 22C11 (1:500; Millipore), anti-HA 3F10 (1:1000; Sigma-Aldrich), anti-myc A14 (1:5000; Santa Cruz Biotechnology), anti-C-terminal DISC1 (1:500; Invitrogen), and IRDye680- and IRDye800-conjugated secondary antibodies (1:10,000; Rockland Immunochemicals), and detected using the LiCor detection system. Immunoprecipitations (IPs) were performed with anti-HAconjugated beads (Sigma-Aldrich) or with C9 and protein A and G (Sigma-Aldrich) and washed in lysis buffer. For endogenous co-IPs, adult rat brains were dounced in Tris-buffered saline with protease inhibitors (Complete; Roche), run through a 27.5 gauge needle, and clarified by centrifugation at $1000 \times g$ for $5 \mathrm{~min}$. Supernatants were centrifuged at $100,000 \times g$ for $1 \mathrm{~h}$ to pellet membranes. Membrane pellet was lysed in 1\% CHAPSO (3- [(3-cholamidopropyl)dimethylammonio]2-hydroxy-1-propanesulfonate) lysis buffer. Immunoprecipitations were performed from both the membrane and cytosolic fractions using C9 or C-terminal DISC1 antibody with a protein A and G mix.

Immunofluorescent staining and confocal microscopy. For analyses of in utero electroporations, paraformaldehyde-fixed brains were washed in PBS, embedded in $2 \%$ agarose, and vibratome sectioned $(150 \mu \mathrm{m})$. Sections were incubated in blocking buffer ( $2 \%$ donkey or goat serum; $0.1 \%$ Triton X-100 in PBS) for $>1 \mathrm{~h}$. For BrdU immunostaining, sections were incubated in $2 \mathrm{~N} \mathrm{HCl}$ for $20 \mathrm{~min}$ at $37^{\circ} \mathrm{C}$ followed by incubation in sodium borate buffer, $\mathrm{pH} 8$, and three PBS washes. Sections were then incubated in primary antibody [anti-microtubule-associated protein 2 (MAP2), 1:10,000 (Abcam); anti-Tbr1, 1:1000 (Abcam); anti-BrdU, 1:100 (Accurate Chemical \& Scientific; Axyll); anti-Ki67, 1:50 (Dako)] between $6 \mathrm{~h}$ and overnight at $4^{\circ} \mathrm{C}$, followed by three washes in PBS. Sections were then incubated with Cy3- and Cy5-conjugated secondary antibodies (1:500-1:1000; Jackson ImmunoResearch) for $>2 \mathrm{~h}$ followed by four PBS washes. Sections were mounted on glass slides using GelMount (Biomeda). Images were acquired using a Zeiss LSM 510 confocal microscope with Axiovert 100M system.

For analyses of DISC1 localization after APP knockdown, primary cortical neurons were plated from Sprague Dawley rats from Charles River and cultured as described previously (Young-Pearse et al., 2008). Neurons were plated onto LabTek CC2 coated two-well chamber slides (Thermo Fisher Scientific) in "plating media" (DMEM plus 5\% fetal calf serum plus penicillin/streptomycin). Four hours after plating, media was changed to Neurobasal medium containing B27 supplement. Twentyfour hours later, neurons were transfected with GFP alone, or with GFP and APP shRNA (Young-Pearse et al., 2007), or else with GFP and a scrambled shRNA (Kamiya et al., 2005) using Lipofectamine 2000 (Invitrogen). Forty-eight hours later, neurons were fixed in $4 \%$ paraformaldehyde, washed with PBS, blocked in $2 \%$ donkey serum plus $0.1 \%$ Triton $\mathrm{X}-100$, and immunostained using either anti-DISC1 [kindly provided by N. Brandon, Wyeth UK, Berkshire, UK (Brandon et al., 2004)] and anti$\gamma$-tubulin (Abcam) or else with anti-DISC1 (Abcam) and antipericentrin (Abcam). Images were acquired using a Zeiss LSM 510 confocal microscope with Axiovert 100M system. GFP-positive (transfected) neurons were categorized as having either a "focal" or "diffuse" pattern of DISC1 immunostaining. All analyses were performed blind to the condition being analyzed. Over 50 cells were analyzed per condition. Data from three independent experiments were acquired and analyzed. Significance was determined using Tukey-Kramer multiplecomparisons tests.

Quantitative analyses of cortical plate entry. For quantitative analyses of migration, all electroporations were performed targeting the same region of the developing cortex. This resulted in a reliable electroporation of the dorsal-lateral region of the neocortex adjacent to the lateral ventricle. After harvest, brains were vibratome sectioned $(100 \mu \mathrm{m})$ in the coronal plane and immunostained for MAP2 to delineate the cortical plate (CP). For each electroporation condition (i.e., each set of electroporated DNAs), more than a total of 500 cells were counted and assessed for their location in either the MAP2 + cortical plate or the intermediate zone (IZ). To determine significant changes relative to control electroporations, at least three independent brains were electroporated and analyzed for each DNA condition. For each independent brain, the percentage of cells in the IZ and CP were calculated. These values were then compared between electroporation conditions using GraphPad InStat. Using this program, one-way ANOVA tests were performed with the Bonferroni multiplecomparisons test. This analysis was used to determine that the percentage of cells in the $\mathrm{CP}$ in the noted electroporation conditions was significantly different from the percentage cells in the $\mathrm{CP}$ of control electroporations, or in the case of rescue experiments, of shRNA-receiving electroporations (similarly, these same conditions were statistically significant when comparing the percentage of cells in the IZ for each condition). 
A

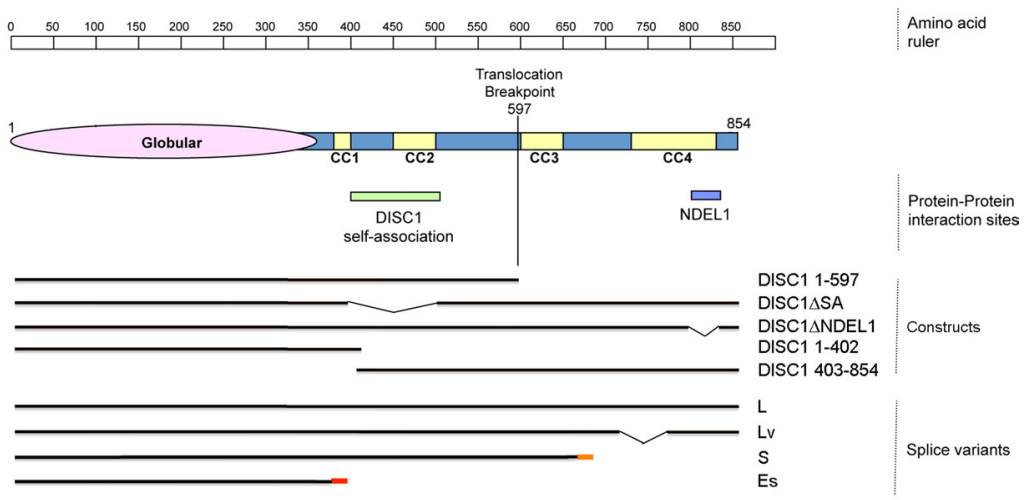

B

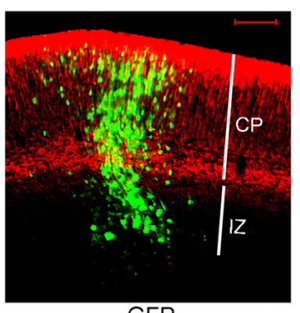

GFP

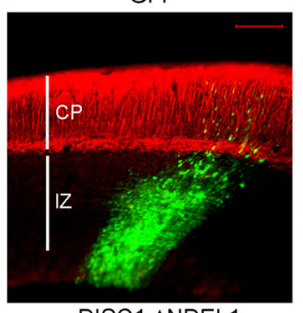

DISC1 $\triangle$ NDEL1

C

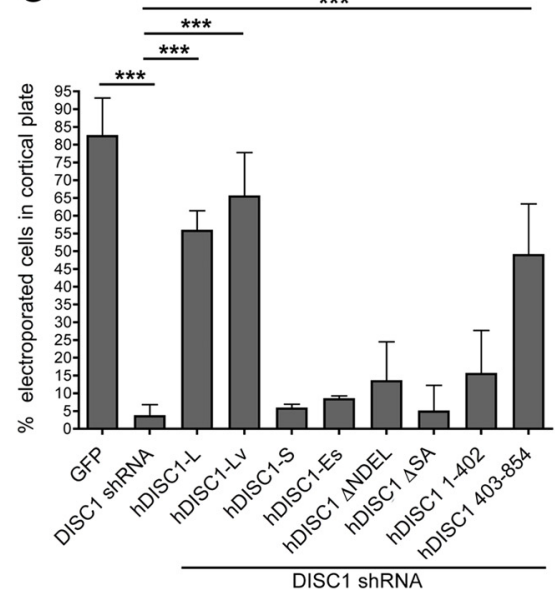

$\mathrm{D}$

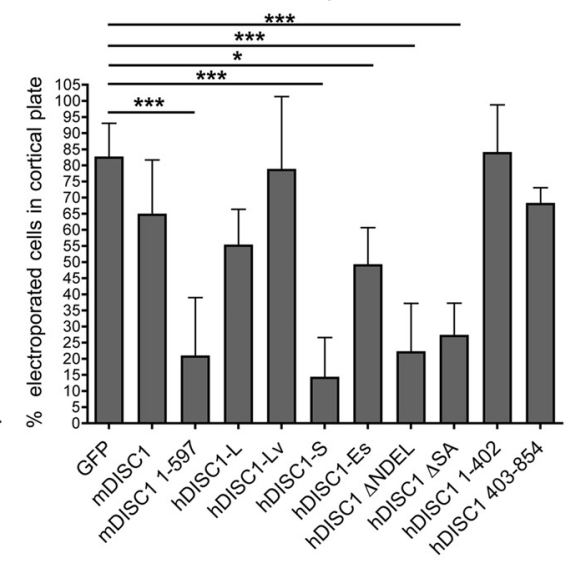

Figure 1. Functional domains of DISC1 involved in cortical migration. $A$, Schematic representation of the DISC1 gene outlining constructs used in this study. $\boldsymbol{B}-\boldsymbol{D}$, E15.5 rat cortices were coelectroporated with GFP and constructs listed. Three days later, brains were fixed, sectioned coronally, and immunostained for MAP2 (red), and the percentage of electroporated cells (green) that migrated into the cortical plate was quantified $(\boldsymbol{C}, \boldsymbol{D})$. Electroporations tested the ability of various DISC1 constructs to rescue the migration defect observed with DISC 1 shRNA $(\boldsymbol{C})$ and also the migration effects of overexpression of each DISC1 construct in the absence of DISC1 shRNA (D). Representative images for some conditions are shown (B). Scale bars, $100 \mu \mathrm{m}$. Data for each construct represent the average from at least three independent brains. Error bars represent $S D .{ }^{*} p<0.05 ;{ }^{* * *} p<0.001$.

\section{Results}

Structure-function relationships of DISC1 in embryonic cortical migration

Previous work has shown that the introduction of DISC1 shRNA by in utero electroporation results in a defect in the ability of neuronal precursor cells to migrate into the cortical plate (Kamiya et al., 2005). In that study, two independent shRNA constructs were used: one that strongly suppressed DISC1 expression and inhibited entry into the cortical plate and a second that had weaker but still significant effects on DISC1 expression and cortical migration (supplemental Fig. $1 A, C$, available at www.jneurosci.org as supplemental material). To further understand the mechanism by which DISC1 exerts its effects on cortical migration, we used in utero electroporation to introduce the stronger DISC1 shRNA construct into cortical precursor cells lining the lateral ventricle of E15.5 rat embryos. Brains were coelectroporated with a GFP construct to follow the fate of the transfected cells. Three days after electroporation, embryonic brains were harvested, fixed, and sectioned coronally. The electroporated sections were immunostained for MAP2 to mark the boundaries of the cortical plate, and the number of transfected (GFP-positive) cells present in different zones of the cortical plate was quantified. When compared with cells electroporated with GFP alone, DISC1 shRNA-electroporated cells displayed a defect in their ability to migrate into the cortical plate, confirming previously published results (Fig. 1B,C) (Kamiya et al., 2005). As a control, a scrambled shRNA did not perturb cortical migration (supplemental Fig. $2 D$, available at www.jneurosci.org as supplemental material). To extend these findings, we performed a series of rescue experiments to examine which regions of DISC1 are necessary and sufficient for its function in migration. Figure $1 A$ outlines the DISC1 protein and the constructs used in this study. Human DISC1 splice variants L (full length) and Lv significantly rescued the DISC1 knockdown migration deficit, whereas the $S$ and Es splice variants, which lack the C-terminal region, did not rescue the phenotype (Fig. 1A-C; supplemental Fig. $2 A, B$, available at www.jneurosci.org as supplemental material). In addition, full-length DISC1 with deletions of either the NDE1/NDEL1 binding site (amino acids 809-854; DISC1 $\Delta$ NDEL1) or the self-association site (amino acids 403504; DISC1 $\Delta$ SA) failed to rescue (Fig. $1 A, C$; supplemental Fig. 2C,G, available at www. jneurosci.org as supplemental material). Interestingly, whereas the N-terminal half of DISC1-L (amino acids 1-402) could not rescue the DISC1 shRNA phenotype, electroporation of the C-terminal half alone (amino acids 403-854) significantly rescued the defect (Fig. 1C; supplemental Fig. 2E,F, available at www.jneurosci.org as supplemental material). 
To characterize further the effects of these DISC1 variants on migration, each DISC1 cDNA was electroporated with GFP but without DISC1 shRNA. In agreement with previous results (Kamiya et al., 2005), overexpression of murine DISC1 lacking C-terminal amino acids corresponding to the translocation breakpoint found in humans (Millar et al., 2000) (mDISC1 1-597) inhibited entry into the cortical plate, whereas overexpression of wild-type DISC1 did not (Fig. $1 B, D$; supplemental Fig. $2 K, L$, available at www.jneurosci.org as supplemental material). In addition, whereas electroporation of DISC1-L and Lv had no effect on normal migration into the cortical plate, electroporation of either DISC1-S or DISC1 with a deletion of the NDEL binding site each resulted in a failure of neuronal precursors to efficiently migrate into the cortical plate (Fig. 1A,B,D; supplemental Fig. $2 H-J$, available at www.jneurosci.org as supplemental material). These two constructs retain amino acids 403-504 and the ability to self-associate (Kamiya et al., 2005). However, DISC1 1-402, which lacks the entire C-terminal half of the protein including this self-interaction site, did not significantly impair cortical plate entry (Fig. 1D). Overexpression of the C-terminal half of DISC1 (403-854), which encodes both a self-association site and the NDEL1 binding site, also did not significantly impair cortical plate entry (Fig. $1 D$; supplemental Fig. $2 M$, available at www.jneurosci.org as supplemental material).

\section{DISC1 acts downstream of APP and}

DAB1 in cortical cell migration

We previously showed that knockdown of APP in neuronal precursor cells prevents proper migration into the cortical plate, using the same in utero paradigm described above (Young-Pearse et al., 2007).

In those experiments, multiple shRNAs targeting APP produced the same phenotype of impaired cortical plate entry (supplemental Fig. $1 B, D$, available at www.jneurosci.org as supplemental material), and full-length human APP cDNA (which is not targeted by the APP shRNAs) was able to fully rescue the defect. The similarities observed between this APP phenotype and that observed for DISC1 (Kamiya et al., 2005) led us to hypothesize that DISC1 may be acting in the same pathway as APP in promoting migration into the cortical plate. To test this, we attempted to rescue DISC1 knockdown with APP and APP knockdown with DISC1 (Fig. 2). Whereas APP coelectroporation with DISC1 shRNA produced no rescue of the latter phenotype, coelectroporation of full-length, wild-type hDISC1-L significantly rescued the APP shRNA phenotype, although the rescue was not complete (Fig. $2 \mathrm{D}$; supplemental Fig. $3 A, C$, available at www.jneurosci.org as supplemental material). Similar to the rescue experiments performed for DISC1 shRNA, electroporation of the C-terminal half of DISC1 (403-
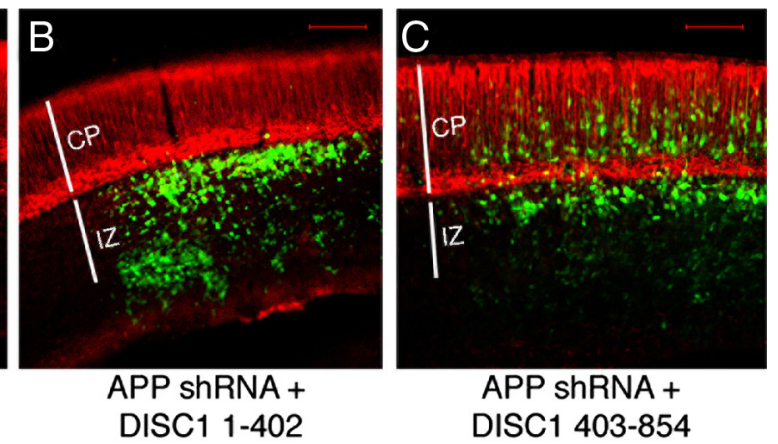

Figure 2. DISC1 acts downstream of APP and DAB1 in cortical migration. E15.5 rat cortices were coelectroporated with GFP and

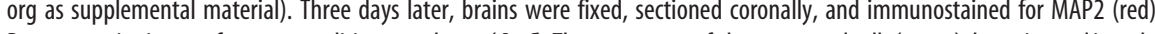
Representative images for some conditions are shown $(\boldsymbol{A}-\boldsymbol{C})$. The percentage of electroporated cells (green) that migrated into the cortical plate was quantified (D). Scale bars, $100 \mu \mathrm{m}$. Data for each construct represents the average from at least three independent brains. Error bars represent SDs. ${ }^{* *} p<0.001$.

854) efficiently rescued the APP shRNA phenotype, whereas expression of the $\mathrm{N}$-terminal half (1-402) had no rescue ability (Fig. $2 B-D$ ).

DAB1 is a cytoplasmic factor well established to play an essential role in cortical cell migration (for review, see Tissir and Goffinet, 2003). We have previously shown that DAB1 acts downstream of APP in cortical cell migration (Young-Pearse et al., 2007). To test whether DISC1 acts downstream of DAB1 or vice versa, we overexpressed DISC1 with DAB1 shRNA or DAB1 with DISC1 shRNA. Whereas DISC1 overexpression significantly rescued the inability of DAB1 shRNA-receiving cells to migrate into the cortical plate (to a similar level that DISC1 had rescued APP knockdown), DAB1 overexpression was unable to provide any rescue of DISC1 shRNA (Fig. 2D; supplemental Fig. 3B,F, available at www.jneurosci.org as supplemental material). Furthermore, DISC1 overexpression rescued the APP knockdown phenotype in the absence of DAB1 (Fig. 2 D; supplemental Fig. $3 G, H$, available at www.jneurosci.org as supplemental material). 


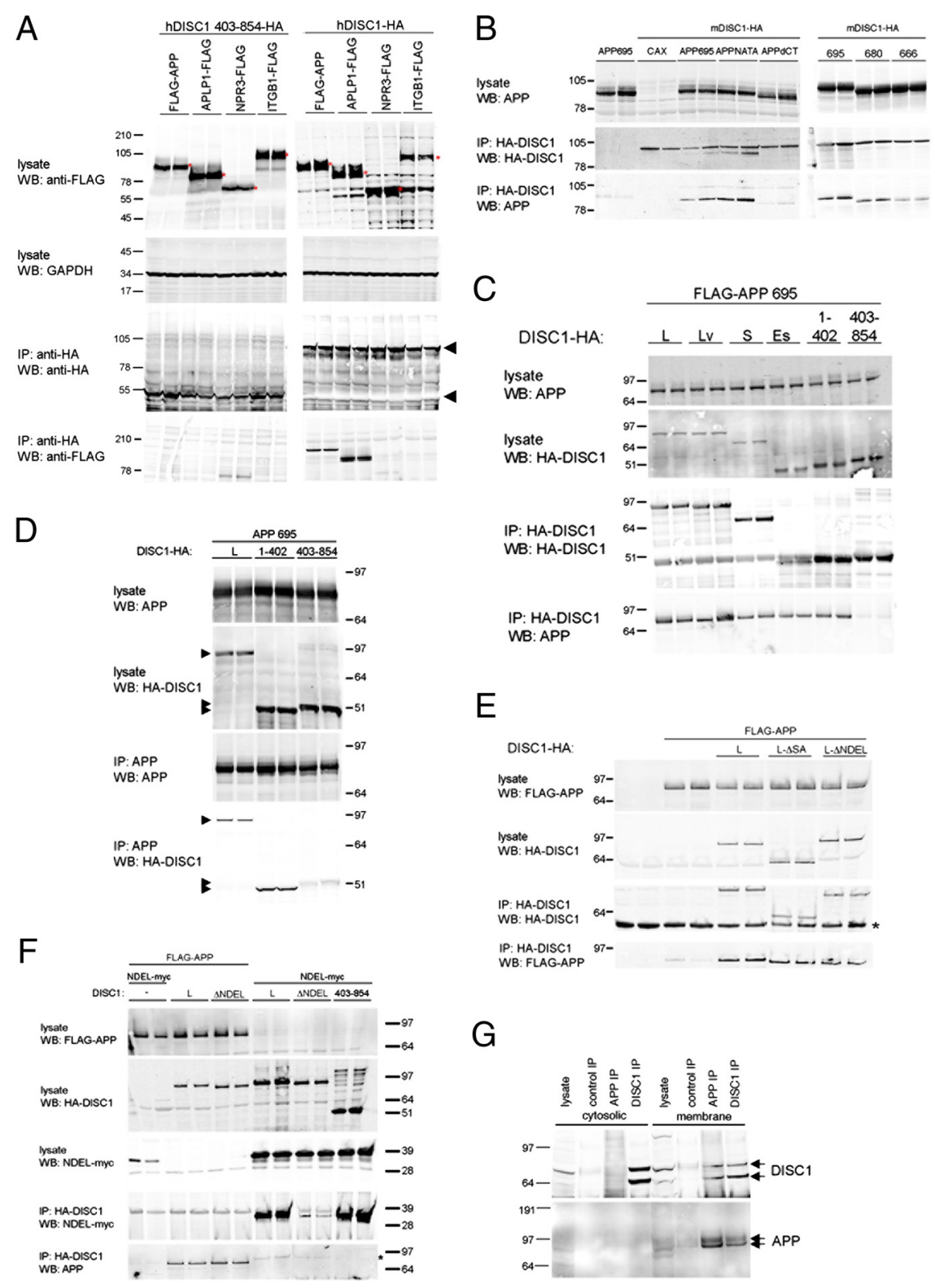

Figure 3. APP and DISC1 interact biochemically. $\operatorname{COS}$ cells were transiently transfected with constructs as listed and harvested $48 \mathrm{~h}$ later in 1\% NP-40 lysis buffer. IPs were performed for HA using 3F10-affinity resin $(\boldsymbol{A}-\boldsymbol{C}, \boldsymbol{E}, \boldsymbol{F})$, or with $22 \mathrm{C11}$ (anti-APP; Millipore) (D). Western blots were run of lysates and IPs as shown. In $\boldsymbol{A}$, the asterisk $\left(^{*}\right)$ denotes relative sizes of each FLAG-tagged protein. $\operatorname{In} \boldsymbol{E}$, the asterisk $\left({ }^{*}\right)$ denotes the $\lg G$ heavy chain band resulting from the immunoprecipitation. $\operatorname{In} \boldsymbol{F}$, the asterisk $\left({ }^{*}\right)$ marks co-IP of endogenous APP with overexpressed DISC1-HA. G, Membrane and cytosolic preps were made from adult rat brains as described in Materials and Methods. Antibodies used for IP were anti-DISC1 (Invitrogen) and anti-APP (C9).

\section{DISC1 and APP interact biochemically}

Through multiple unbiased screens for DISC1 interacting proteins, $>100$ candidate DISC1 interactors have been identified (Millar et al., 2003; Brandon, 2007; Camargo et al., 2007). One of the proteins identified was APLP1 (Millar et al., 2003), an APP family member. Because of the high sequence homology between APLP1 and its family members in their intracellular domains, we sought to examine whether APP family members and DISC1 exist in a complex with one another. COS cells were transiently transfected with HA-tagged human DISC1 plus one of several FLAGtagged type I transmembrane proteins, including APP, APLP1, natriuretic peptide receptor 3 (NPR3), or integrin $\beta 1$ (ITGB1), the latter two were used as negative controls. Cells were harvested with 1\% NP-40 lysis buffer $48 \mathrm{~h}$ later. DISC1 was pulled down with anti-HA resin, and anti-FLAG Western blots were performed to examine the ability of DISC1 to interact with each of these four transmembrane proteins. DISC1 pulled down FLAG-tagged APP and APLP1, whereas it was unable to pull down FLAG-tagged ITGB1 or NPR3 (Fig. $3 A)$. Because DISC1 is an intracellular protein, we anticipated that it would interact with the C-terminal domain of APP. We found that DISC1 efficiently pulled down full-length APP, whereas deletion of the C-terminal domain of APP $(\triangle \mathrm{CT})$ essentially eliminated this coimmunoprecipitation (Fig. 3B).

Within the intracellular domain of APP, the NPTY domain is responsible for interacting with all of the known intracellular binding partners of APP, including DAB1, Fe65, and the MINT family (Rogelj et al., 2006). To determine whether the APP NPTY domain is necessary for the interaction with DISC1, we mutated this domain (to NATA) and performed transfection and co-IP experiments as above. Mutation of the NPTY motif within APP did not abrogate the co-IP between APP and DISC1 (Fig. 3B). To narrow down the site of DISC1 interaction within APP, we deleted the last 15 aa (APP 680) or the last 29 aa (APP 666) of the 50-residue C-terminal intracellular domain of APP. Although APP 680 still efficiently coimmunoprecipitated with DISC1, APP 666 showed markedly decreased coimmunoprecipitation (Fig. 3B), suggesting that residues critical for the DISC1 interaction reside in these 14 aa of APP (666-680). As mutation (NPTY $\rightarrow$ NATA) or deletion (APP 680) of the DAB1 interaction site within (NPTY $\rightarrow$ NATA) did not alter DISC1 interaction, we next examined whether DAB1 can exist in a complex with DISC1. To this end, we performed coimmunoprecipitation experiments between DAB1, APP, and DISC1. As expected, APP co-IPed with both DAB1 and DISC1 (supplemental Fig. 4, available at www.jneurosci. org as supplemental material). Furthermore, DISC1 co-IPed DAB1 in the presence and absence of overexpressed APP (supplemental Fig. 4, available at www.jneurosci.org as supplemental material). Although the co-IP between DISC1 and DAB1 could be dependent on the presence of endogenous APP, these results suggest that DAB1 can exist in a complex with both APP and DISC1.

Additional analysis revealed that APP can co-IP with all four splice variants of DISC1 but not with a C-terminal fragment (amino acids 403-854) of DISC1 (Fig. 3C). The apparent interaction between APP and the N-terminal half of DISC1 was confirmed by performing the reverse IP: when APP was immunoprecipitated with an antibody to its $\mathrm{N}$ terminus, full-length DISC1 and DISC1 1-402 were pulled down, whereas DISC1 403854 was not (Fig. 3D). Deletion of the DISC1 self-association site 
(DISC1 $\Delta$ SA) did not affect DISC1-APP co-IP (Fig. 3E). In addition, although deletion of the NDEL binding site (DISC1 1 NDEL1) eliminated coimmunoprecipitation of DISC1 and NDEL as expected (Fig. $3 F$ ), it did not affect the co-IP of DISC1 and APP (Fig. 3E, F). Figure $3 F$ also shows that exogenous HA-DISC1-L can co-IP endogenously expressed APP, whereas HA-DISC1 403-854 cannot (see asterisk). Finally, Figure $3 G$ shows that DISC1 and APP expressed at endogenous levels in normal rat brain tissue coimmunoprecipitate. DISC1 is localized to multiple subcellular compartments, with a small portion present in membrane fractions (Fig. $3 G)$, leading us to expect that an endogenous interaction between DISC1 and APP in neurons may be transient. Since APP is a type I transmembrane domain protein, we performed coimmunoprecipitations for DISC1 and APP from a membrane fraction of adult rat brain to enrich for this complex. Although co-IP was not observed in the cytosolic fraction in which full-length APP is not present, DISC1 was pulled down with APP in the membrane fraction (Fig. 3G).

\section{Knockdown of APP in cortical neurons} does not affect cell cycle entry at E15.5

Recently, Mao et al. (2009) showed that acute knockdown of DISC1 in younger neuronal precursor cells (E13) in the cortex reduces their proliferation. Although these studies did not show an inhibition of migration into the cortical plate with DISC1 knockdown at this earlier developmental age, the same laboratory has recently observed a defect in migration into the cortical plate after DISC1 knockdown at later embryonic stages (K. Singh and L.-H. Tsai, personal communication). To test whether the defects observed with DISC1 or APP knockdown could be related to the role of DISC1 in proliferation, we electroporated E15.5 embryonic brains, injected BrdU $24 \mathrm{~h}$ later, and harvested $24 \mathrm{~h}$ after BrdU injection. Brains were then fixed, sectioned coronally, and immunostained using an antibody for BrdU (Fig. 4A-C). Forty-eight hours after electroporation, a significant inhibition of migration into the cortical plate was observed with knockdown of APP or DISC1 (Fig. 4D), as expected from our previous results (above). As had been observed at $72 \mathrm{~h}$ after electroporation (Fig. 2D), APP and DISC1 were each able to significantly rescue APP knockdown at $48 \mathrm{~h}$ (Fig. $4 \mathrm{D}$ ). In fact, rescue with DISC1 appeared to be more effective at this earlier time point. This may be attributable to one or more of several reasons, including levels of overexpression of the DISC1 rescue construct or lower effects of APP shRNA at $48 \mathrm{~h}$ relative to $72 \mathrm{~h}$. Although APP knockdown resulted in a significant inhibition of migration into the cortical plate at this $48 \mathrm{~h}$ time point (Fig. $4 \mathrm{D}$ ), neither knockdown of APP nor of DISC1 had a significant effect on BrdU incorporation in cells in the ventricular zone (VZ)/subventricular zone (SVZ)/IZ at this time point (Fig. $4 E$ ).

\section{Knockdown of APP in cortical neurons results in DISC1 mislocalization}

In view of the above evidence of both genetic and biochemical interactions between DISC1 and APP, we investigated whether changes in APP affect DISC1 expression and/or subcellular localization. DISC1 protein levels were not significantly altered by the loss of APP expression in APP knock-out brain lysates (supplemental Fig. 5, available at www.jneurosci.org as supplemental material). To investigate whether APP knockdown affects DISC1 subcellular localization, GFP was cotransfected into rat cortical neurons with either control shRNA or APP shRNA or else was electroporated alone. Seventy-two hours later, neurons were fixed and immunostained for DISC1 using two different antibodies (Fig. 5; supplemental Fig. 6, available at www.jneurosci.org as supplemental material). The majority of neurons transfected with GFP alone (Fig. 5A, $B$; supplemental Fig. $6 A-C$, available at www.jneurosci.org as supplemental material) or with GFP plus control shRNA (Fig. 5D-F; supplemental Fig. 6D-F, available at www.jneurosci.org as supplemental material) displayed a principally focal pattern of intense DISC1 immunoreactivity within the neurons, accompanied by some more diffuse immunostaining. In contrast, neurons transfected with GFP plus APP shRNA showed a change in the pattern of DISC1 immunolocalization to a principally diffuse staining throughout the perikaryon (Fig. $5 G-I$; supplemental Fig. $5 G-I$, available at www.jneurosci.org as supplemental material). To quantify these effects on DISC1 subcellular localization, immunostained cultures were analyzed blinded to treatment, and individual GFP-positive transfected neurons were scored for the presence of DISC1 immunoreactivity as either focal or diffuse. Knockdown of APP resulted in a significant shift in DISC1 subcellular localization from a principally focal to a diffuse pattern (Fig. 5J; supplemental Fig. $6 \mathrm{~J}$, available at www.jneurosci.org as supplemental material). This shift in localization was rescued by cotransfection with human wild-type APP but not human APP lacking its C terminus (Fig. 5J; supplemental Fig. 7, available at www.jneurosci.org as supplemental material). In this regard, it has been shown that the func- 


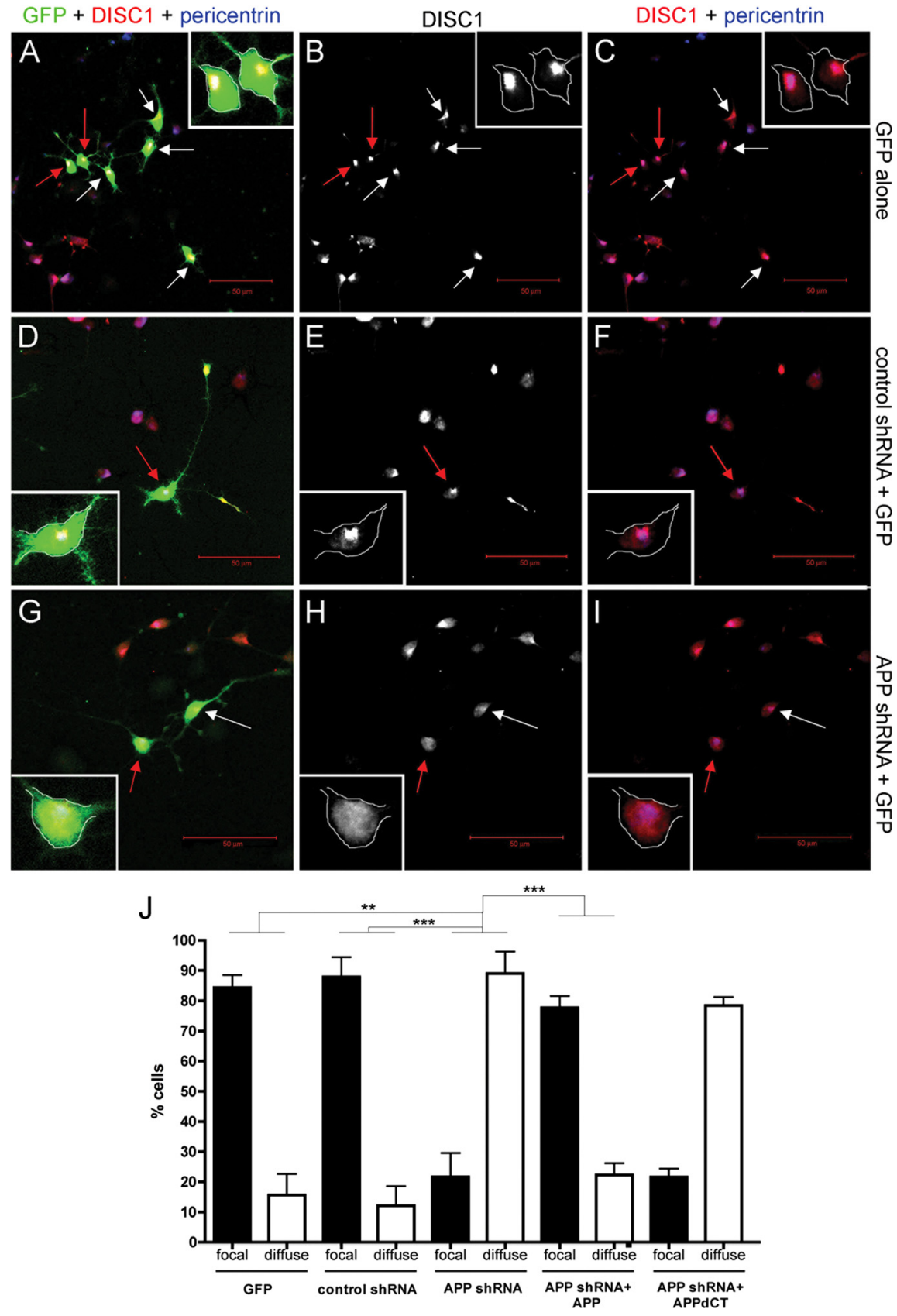

Figure 5. APP knockdown results in altered DISC1 localization in cortical neurons. Primary rat cortical neurons were cotrans fected with GFP alone or with GFP plus control shRNA or APP shRNA constructs. Three days later, neurons were fixed and immunostained for DISC1 (Abcam) (shown in red in $\boldsymbol{A}, \boldsymbol{C}, \boldsymbol{D}, \boldsymbol{F}, \boldsymbol{G}, \boldsymbol{I}$; or white in $\boldsymbol{B}, \boldsymbol{E}, \boldsymbol{H}$ ) and pericentrin (blue). Confocal images were acquired, and representative images are shown $(\boldsymbol{A}-\boldsymbol{I})$. The insets show magnified views of the cells indicated by red arrows. Identities of the images were deidentified, and DISC1 localization was examined in GFP-positive cotransfected cells (indicated by white arrows). Cells were classified as having focal ( $\boldsymbol{B}, \boldsymbol{E}$, arrows) or diffuse DISC1 ( $\boldsymbol{H}$, arrow) immunostaining. Data from three independent experiments are shown in $J$. At least 50 cells were quantified for each condition. Error bars represent SDs between the three experiments. ${ }^{* *} p<0.01 ;{ }^{* *} p<0.001$

tion of DISC1 in cortical cell migration requires DISC1 to localize to the centrosome (Kamiya et al., 2005). To test whether the focal pattern observed for DISC1 localization in normal (untreated) cells could reflect its localization to the centrosome, we coimmunolabeled the transfected cortical neurons for DISC1 and either pericentrin (Fig. 5C, F, I) or $\gamma$-tubulin (supplemental Fig. 6C, F, $I$, available at www.jneurosci.org as supplemental material). The focal DISC1 immunoreactivity observed in control transfections colocalized with the immunoreactivity for these centrosomal markers. Together, our results are consistent with the hypothesis that DISC1 localizes in part to the centrosome and that knockdown of APP decreases this localization in embryonic cortical neurons and prevents their proper entry into the cortical plate.

\section{Discussion}

\section{Identification of DISC1 functional} domains involved in cortical migration Acute deficiency of DISC1 in cortical precursor cells at embryonic day 15.5 results in a failure of migrating neuronal precursors to enter the cortical plate (Fig. 1) (Kamiya et al., 2005). As described above, it was recently shown that acute knockdown of DISC1 in younger neuronal precursor cells (E13) in the cortex reduces their proliferation (Mao et al., 2009). Here, we do not observe a significant alteration in proliferation (BrdU incorporation) using the paradigm of APP or DISC1 knockdown at E15.5, BrdU injection at E16.5, and harvest at E17.5 (Fig. 4). However, we confirm a migration defect using this protocol. These results suggest that the roles for APP and DISC1 in proliferation and migration are separable and that the APP knockdown migration phenotype is not an indirect effect of proliferation defects.

In the studies presented here, we performed detailed analyses to examine which splice variants and regions of DISC1 are critical for its function in neuronal precursor cells at relatively late embryonic time points (E16-E18), as assayed by cell entry into the cortical plate. In humans, multiple endogenously expressed splice variants have been identified. We show that, although hDISC1-L (full length) and -Lv efficiently rescue the DISC1 knockdown migration phenotype, the $-S$ and -Es splice variants provide no rescue ability. As schematized in Figure $1 A$, the -S and -Es variants lack the proposed NDE1/NDEL1 binding site, and deletion of this binding site alone eliminates the ability of DISC1 to rescue migration. Furthermore, we confirmed previous results (Kamiya et al., 2005) that showed a dominant-negative effect of overexpressing hDISC1 bearing a C-terminal truncation that corresponds to the known translocation breakpoint associated with schizophrenia. Similarly, overexpressing hDISC1-S, which lacks the NDE1/NDEL1 binding site, also acts as a dominant negative. In contrast, overexpression of a construct encoding just the first 402 aa of hDISC1, which lacks both the NDE1/NDEL1 binding site and a self-association site, does not alter the normal migration of neurons into the cortical plate. These results support the hypoth- 


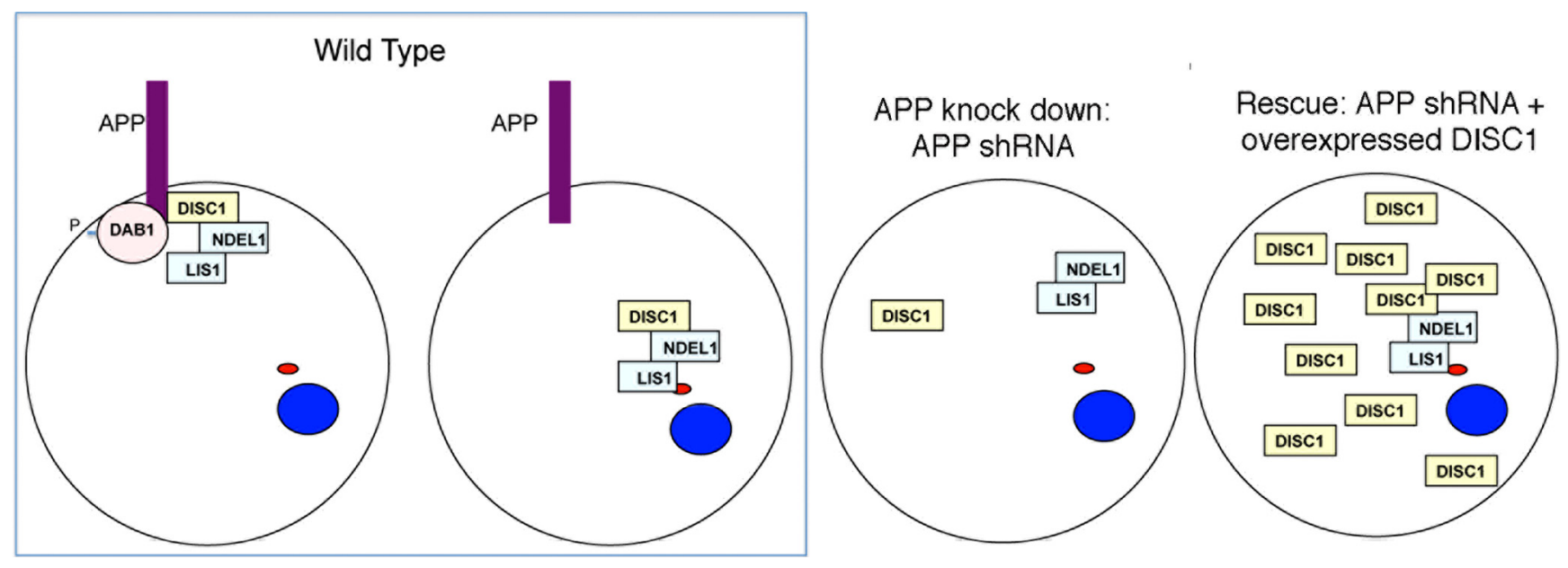

Figure 6. Summary of data showing APP and DISC1 interactions in cortical cell migration. In wild-type cells, APP exists in a complex with DAB1 and DISC1 through distinct binding domains in its cytoplasmic region. In addition to interacting with APP at the cell surface and/or on intracellular membranes, DISC1 localizes to the centrosome with its binding partners NDEL1 and LIS1, where it carries out its role in nucleokinesis (Morris et al., 2003; Kamiya et al., 2005; Bradshaw et al., 2008). When APP is knocked down, DISC1 is localized more diffusely throughout the cytoplasm and cannot perform its function in migration. However, when cells lacking APP are flooded with excess DISC1, the need for the APP-DISC1 biochemical interaction is bypassed and the migration defect is rescued. The small red ellipse represents the centrosome, and the blue oval represents the nucleus.

esis that overexpressed DISC1 lacking the far C terminus but retaining the self-association site can act as a dominant negative by binding to and "inactivating" endogenous DISC1, which would normally bind NDEL1 and thus LIS1. In direct support of the hypothesis that DISC1 interacts with itself to mediate its function in migration, deletion of the self-association site alone eliminates the ability of hDISC1 to rescue DISC1 knockdown. Finally, we show that overexpressing the C-terminal half alone (but not the N-terminal half alone) of hDISC1-L efficiently rescues the impaired migration resulting from DISC1 knockdown. Thus, when overexpressed, the N-terminal domain of DISC1 [which is critical for proliferation (Mao et al., 2009)] is dispensable for the function of DISC1 in migration. This supports the finding that the apparently later function of DISC1 in cortical plate entry of precursors can be separated from its earlier developmental role in precursor proliferation.

\section{DISC1 acts downstream of APP and DAB1 in cortical precursor cell migration}

We have previously shown that acute knockdown of APP in cortical precursor cells results in a migration defect that appears indistinguishable from that observed with DISC1 knockdown (Young-Pearse et al., 2007) (Figs. 1B, 2A). Similar to DISC1 knockdown (Kamiya et al., 2005), the majority of cells that lack APP are able to migrate out of the ventricular zone but fail to enter the cortical plate, being retained in the intermediate zone (Young-Pearse et al., 2007). Furthermore, DAB1 knockdown via in utero electroporation produces a similar phenotype, and DAB1 overexpression partially rescues the APP knockdown phenotype (Young-Pearse et al., 2007). Although it is well established that DAB1 binds to the NPTY motif in the cytoplasmic C-terminal domain of APP (Homayouni et al., 1999; Howell et al., 1999; Trommsdorff et al., 1999), the mechanism by which DAB1 functions downstream of APP is unclear. Here, we present evidence that hDISC1 can significantly rescue both the APP and DAB1 knockdown migration phenotypes. In contrast, neither APP nor DAB1 overexpression provides rescue of the DISC1 knockdown phenotype. These data lead us to conclude that DISC1 acts down- stream of APP and DAB1 to regulate proper neuronal migration into the cortical plate.

Previous studies have identified many DISC1 interacting factors (for review, see Brandon, 2007), including APLP1 (Millar et al., 2003), an APP family member having 50\% amino acid identity with APP in their C-terminal intracellular domains. We confirmed here that APLP1 and DISC1 interact using coimmunoprecipitation experiments and went on to show that APP and DISC1 interact through the C-terminal intracellular domain of APP and the $\mathrm{N}$-terminal domain of DISC1. The NPTY domain of APP is required for the interaction of APP with each of its previously known intracellular binding proteins (Fe65, DAB1, MINTs) (Homayouni et al., 1999; Howell et al., 1999; Trommsdorff et al., 1999). However, we show that DISC1 still interacts with APP when this site is mutated or deleted, indicating that DAB1 and DISC1 have distinct binding sites within the APP intracellular domain. Moreover, we find that APP and NDE1/NDEL1 have distinct binding sites within DISC1. Therefore, the interactions of each of these binding partners need not be mutually exclusive.

\section{Loss of APP affects DISC1 localization in embryonic neurons}

DISC1 was previously shown to localize to multiple subcellular compartments, depending on the cell type and developmental stage investigated. DISC1 has been shown to localize in part to the centrosome in neuronal precursor cells and mature neurons (Morris et al., 2003; Ozeki et al., 2003; Kamiya et al., 2005; Bradshaw et al., 2008). Because DISC1 centrosomal localization is crucial for migration (Kamiya et al., 2005) and overexpression of DISC1 rescues APP knockdown, we hypothesized that loss of APP in embryonic neurons may affect DISC1 subcellular localization. We find that primary cortical neurons that are transfected with GFP alone or with a control scrambled shRNA construct display an intense focal pattern of DISC1 immunofluorescence, as well as some diffuse staining, in the cell body. The principally focal localization of DISC1 is observed with two independent DISC1 antibodies and colocalizes with pericentrin and $\gamma$-tubulin, two proteins present at centrosomes. Importantly, knockdown of APP results in a loss of this focal DISC1 immuno- 
staining and an increase in diffuse cytoplasmic immunostaining. These results support the hypothesis that APP helps regulate proper DISC1 subcellular localization in immature cortical neurons.

A critical next step will be to determine which subcellular pool of APP is important for DISC1 localization. We previously showed that the uncleaved APP holoprotein is required for proper neuronal migration, and we proposed that the interaction of holoAPP at the cell surface with extracellular cues would regulate the migration of neuronal precursor cells past the cortical plate boundary (Young-Pearse et al., 2007). However, in addition to its cell surface localization, APP has been reported to localize in part to the centrosome in $\mathrm{H} 4$ neuroglioma cells (Nizzari et al., 2007). In that study, a loss of APP affected the translocation of ERK1/2 to the centrosome, similar to the results that we show here for DISC1. Although still controversial (Lazarov et al., 2005), it has been proposed that APP affects motor proteinassociated transport in neurons through its interaction with kinesin (Kamal et al., 2000; Gunawardena and Goldstein, 2001), suggesting a possible mechanism by which a loss of APP could affect DISC1 translocation to the centrosome.

\section{Summary model of APP-DISC1 functional and biochemical interaction in the migration of neuronal precursor cells into the cortical plate}

To summarize (Fig. 6), our results suggest that, in neurons, a subset of DISC1 exists in a complex with APP in a membrane fraction (Fig. $3 G$ ), whereas the majority is present at the centrosome (Fig. 5). Although the N terminus of DISC1 is responsible for interaction with APP (Fig. 3), the far C terminus binds to the NDEL1/LIS1 complex (Ozeki et al., 2003; Brandon et al., 2004; Kamiya et al., 2006). This DISC1/NDEL1/LIS1 complex was previously shown to localize to the centrosome and this localization is well established to be required for proper nucleokinesis and therefore migration (Feng et al., 2000; Brandon et al., 2004; Tanaka et al., 2004). When APP is knocked down in embryonic neurons, DISC1 becomes diffusely localized throughout the perikaryon (Fig. 5), and these cells cannot properly migrate into the cortical plate (Fig. 2). In contrast, overexpressing DISC1 rescues the defect in migration observed with APP knockdown (Fig. 2 ). We speculate that, by flooding the cell with DISC1, a sufficient portion is able to localize to the centrosome, bypassing the need for its physical interaction with APP. In support of this hypothesis, overexpressing a form of DISC1 lacking its APP binding domain rescues both the APP knockdown and DISC1 knockdown migration phenotypes (Figs. 1,2).

In conclusion, we present evidence that functionally and biochemically links APP and DISC1, two genes critically involved in very different disorders of the human cerebral cortex. Elucidating the mechanism by which these gene products and their partners integrate into common pathways to mediate cortical development and function is an important step in understanding how genetic alterations in each protein lead to disparate pathologies.

\section{References}

Beecham GW, Martin ER, Li YJ, Slifer MA, Gilbert JR, Haines JL, PericakVance MA (2009) Genome-wide association study implicates a chromosome 12 risk locus for late-onset Alzheimer disease. Am J Hum Genet 84:35-43.

Bradshaw NJ, Ogawa F, Antolin-Fontes B, Chubb JE, Carlyle BC, Christie S, Claessens A, Porteous DJ, Millar JK (2008) DISC1, PDE4B, and NDE1 at the centrosome and synapse. Biochem Biophys Res Commun 377:1091-1096.

Brandon NJ (2007) Dissecting DISC1 function through protein-protein interactions. Biochem Soc Trans 35:1283-1286.
Brandon NJ, Handford EJ, Schurov I, Rain JC, Pelling M, Duran-Jimeniz B, Camargo LM, Oliver KR, Beher D, Shearman MS, Whiting PJ (2004) Disrupted in schizophrenia 1 and Nudel form a neurodevelopmentally regulated protein complex: implications for schizophrenia and other major neurological disorders. Mol Cell Neurosci 25:42-55.

Camargo LM, Collura V, Rain JC, Mizuguchi K, Hermjakob H, Kerrien S, Bonnert TP, Whiting PJ, Brandon NJ (2007) Disrupted in schizophrenia 1 interactome: evidence for the close connectivity of risk genes and a potential synaptic basis for schizophrenia. Mol Psychiatry 12:74-86.

De Strooper B, Annaert W (2000) Proteolytic processing and cell biological functions of the amyloid precursor protein. J Cell Sci 113:1857-1870.

Feng Y, Olson EC, Stukenberg PT, Flanagan LA, Kirschner MW, Walsh CA (2000) LIS1 regulates CNS lamination by interacting with mNudE, a central component of the centrosome. Neuron 28:665-679.

Gunawardena S, Goldstein LS (2001) Disruption of axonal transport and neuronal viability by amyloid precursor protein mutations in Drosophila. Neuron 32:389-401.

Hemming ML, Elias JE, Gygi SP, Selkoe DJ (2008) Proteomic profiling of gamma-secretase substrates and mapping of substrate requirements. PLoS Biol 6:e257.

Homayouni R, Rice DS, Sheldon M, Curran T (1999) Disabled-1 binds to the cytoplasmic domain of amyloid precursor-like protein 1. J Neurosci 19:7507-7515.

Howell BW, Lanier LM, Frank R, Gertler FB, Cooper JA (1999) The disabled 1 phosphotyrosine-binding domain binds to the internalization signals of transmembrane glycoproteins and to phospholipids. Mol Cell Biol 19:5179-5188.

Ishizuka K, Paek M, Kamiya A, Sawa A (2006) A review of disrupted-inschizophrenia-1 (DISC1): neurodevelopment, cognition, and mental conditions. Biol Psychiatry 59:1189-1197.

Jaaro-Peled H, Hayashi-Takagi A, Seshadri S, Kamiya A, Brandon NJ, Sawa A (2009) Neurodevelopmental mechanisms of schizophrenia: understanding disturbed postnatal brain maturation through neuregulin-1-ErbB4 and DISC1. Trends Neurosci 32:485-495.

Kamal A, Stokin GB, Yang Z, Xia CH, Goldstein LS (2000) Axonal transport of amyloid precursor protein is mediated by direct binding to the kinesin light chain subunit of kinesin-I. Neuron 28:449-459.

Kamiya A, Kubo K, Tomoda T, Takaki M, Youn R, Ozeki Y, Sawamura N, Park U, Kudo C, Okawa M, Ross CA, Hatten ME, Nakajima K, Sawa A (2005) A schizophrenia-associated mutation of DISC1 perturbs cerebral cortex development. Nat Cell Biol 7:1167-1178.

Kamiya A, Tomoda T, Chang J, Takaki M, Zhan C, Morita M, Cascio MB, Elashvili S, Koizumi H, Takanezawa Y, Dickerson F, Yolken R, Arai H, Sawa A (2006) DISC1-NDEL1/NUDEL protein interaction, an essential component for neurite outgrowth, is modulated by genetic variations of DISC1. Hum Mol Genet 15:3313-3323.

Lazarov O, Morfini GA, Lee EB, Farah MH, Szodorai A, DeBoer SR, Koliatsos VE, Kins S, Lee VM, Wong PC, Price DL, Brady ST, Sisodia SS (2005) Axonal transport, amyloid precursor protein, kinesin-1, and the processing apparatus: revisited. J Neurosci 25:2386-2395.

Mao Y, Ge X, Frank CL, Madison JM, Koehler AN, Doud MK, Tassa C, Berry EM, Soda T, Singh KK, Biechele T, Petryshen TL, Moon RT, Haggarty SJ, Tsai LH (2009) Disrupted in schizophrenia 1 regulates neuronal progenitor proliferation via modulation of GSK3beta/beta-catenin signaling. Cell 136:1017-1031.

Millar JK, Wilson-Annan JC, Anderson S, Christie S, Taylor MS, Semple CA, Devon RS, St Clair DM, Muir WJ, Blackwood DH, Porteous DJ (2000) Disruption of two novel genes by a translocation co-segregating with schizophrenia. Hum Mol Genet 9:1415-1423.

Millar JK, Christie S, Porteous DJ (2003) Yeast two-hybrid screens implicate DISC1 in brain development and function. Biochem Biophys Res Commun 311:1019-1025.

Miyoshi K, Honda A, Baba K, Taniguchi M, Oono K, Fujita T, Kuroda S, Katayama T, Tohyama M (2003) Disrupted-in-schizophrenia 1, a candidate gene for schizophrenia, participates in neurite outgrowth. Mol Psychiatry 8:685-694.

Morris JA, Kandpal G, Ma L, Austin CP (2003) DISC1 (disrupted-inschizophrenia 1) is a centrosome-associated protein that interacts with MAP1A, MIPT3, ATF4/5 and NUDEL: regulation and loss of interaction with mutation. Hum Mol Genet 12:1591-1608.

Nizzari M, Venezia V, Repetto E, Caorsi V, Magrassi R, Gagliani MC, Carlo P, Florio T, Schettini G, Tacchetti C, Russo T, Diaspro A, Russo C (2007) 
Amyloid precursor protein and Presenilin1 interact with the adaptor GRB2 and modulate ERK 1,2 signaling. J Biol Chem 282:13833-13844.

Ozeki Y, Tomoda T, Kleiderlein J, Kamiya A, Bord L, Fujii K, Okawa M, Yamada N, Hatten ME, Snyder SH, Ross CA, Sawa A (2003) Disruptedin-schizophrenia-1 (DISC-1): mutant truncation prevents binding to NudE-like (NUDEL) and inhibits neurite outgrowth. Proc Natl Acad Sci U S A 100:289-294.

Rogelj B, Mitchell JC, Miller CC, McLoughlin DM (2006) The X11/Mint family of adaptor proteins. Brain Res Rev 52:305-315.

Tanaka T, Serneo FF, Higgins C, Gambello MJ, Wynshaw-Boris A, Gleeson JG (2004) Lis1 and doublecortin function with dynein to mediate coupling of the nucleus to the centrosome in neuronal migration. J Cell Biol 165:709-721.
Tissir F, Goffinet AM (2003) Reelin and brain development. Nat Rev Neurosci 4:496-505.

Trommsdorff M, Gotthardt M, Hiesberger T, Shelton J, Stockinger W, Nimpf J, Hammer RE, Richardson JA, Herz J (1999) Reeler/Disabled-like disruption of neuronal migration in knockout mice lacking the VLDL receptor and ApoE receptor 2. Cell 97:689-701.

Young-Pearse TL, Bai J, Chang R, Zheng JB, LoTurco JJ, Selkoe DJ (2007) A critical function for $\beta$-amyloid precursor protein in neuronal migration revealed by in utero RNA interference. J Neurosci 27: 14459-14469.

Young-Pearse TL, Chen AC, Chang R, Marquez C, Selkoe DJ (2008) Secreted APP regulates the function of full-length APP in neurite outgrowth through interaction with integrin beta1. Neural Dev 3:15. 